DEMONSTRATIO MATHEMATICA

Vol. XXXIII No $3 \quad 2000$

M. K. Aouf, H. E. Darwish, A. E. Alhosseny

\title{
A GENERALIZATION OF $p$-VALENT CLASSES RELATED TO CONVEX FUNCTIONS
}

Abstract. In this paper we shall study some properties of the class $C(b, p, A, B)$, where $C(b, p, A, B)$ is defined as follows :

$$
p+\frac{1}{b}\left(1+\frac{z f^{\prime \prime}(z)}{f^{\prime}(z)}-p\right) \prec p \frac{1+A z}{1+B z}, z \in U,
$$

where $\prec$ denote subordination, $b \neq 0$ complex, $p \in I N$ and $-1 \leq B<A \leq 1$.

\section{Introduction}

Let $A_{p}(p \in N)$ denote the class of functions of the form

$$
f(z)=z^{p}+\sum_{k=p+1}^{\infty}(p \in N)
$$

which are analytic and $p$-valent in the unit disc $U=\{z:|z|<1\}$.

For $|\lambda|<\frac{\pi}{2}$ and $p$ a fixed positive integer, let $S_{p}^{\lambda}(\alpha)$ denote the class of functions $f(z) \in A_{p}$ which satisfy the inequality

$$
\operatorname{Re}\left\{e^{i \lambda} \frac{z f^{\prime}(z)}{f^{\prime}(z)}\right\}>\alpha \cos \lambda \quad(z \in U),
$$

for some $\alpha(0 \leq \alpha<p)$. Following Patil and Thakare [9], we say that the functions belonging to the class $S_{p}^{\lambda}(\alpha)$ are $p$-valent $\lambda$-spiral-like functions of order $\alpha$.

Furthermore, for $|\lambda|<\frac{\pi}{2}$ and $p$ a fixed positive integer, let $C_{p}^{\lambda}(\alpha)$ denote the class of functions $f(z) \in A_{p}$ which satisfy the inequality

$$
\operatorname{Re}\left\{e^{i \lambda}\left(1+\frac{z f^{\prime \prime}(z)}{f^{\prime}(z)}\right)\right\}>\alpha \cos \lambda, \quad z \in U
$$

1991 Mathematics Subject Classification: 30C45.

Key words and phrases: convex, p-valent. 
for some $\alpha(0 \leq \alpha<p)$. We say that the functions belonging to the class $C_{p}^{\lambda}(\alpha)$ are $p$-valent $\lambda$-Robertson functions of order $\alpha$.

It follows from (1.2) and (1.3) that

$$
f(z) \in C_{p}^{\lambda}(\alpha) \quad \text { if and only if } \frac{z f^{\prime}(z)}{p} \in S_{p}^{\lambda}(\alpha) .
$$

Definition 1 [1]. A function $f(z) \in A_{p}$ is said to be $p$-valent convex function of order $b$ ( $b \neq 0$ complex), that is $f(z) \in C(b, p)$ if and only if

$$
\operatorname{Re}\left\{p+\frac{1}{b}\left(1+\frac{z f^{\prime \prime}(z)}{f^{\prime}(z)}-p\right)\right\}>0, \quad z \in U
$$

For $b \neq 0$ complex, $p \in N$, and $-1 \leq B<A<1$, let $C(b, p, A, B)$ denote the class of functions of $A_{p}$ which satisfy the condition

$$
p+\frac{1}{b}\left(1+\frac{z f^{\prime \prime}(z)}{f^{\prime}(z)}-p\right) \prec \frac{1+A z}{1+B z}, \quad z \in U .
$$

In other words $f(z) \in C(b, p, A, B)$ if and only if there exists a function $w(z) \in \Omega$ such that

$$
\frac{z f^{\prime \prime}(z)}{f^{\prime}(z)}=\frac{(p-1)+[(A-B) p b+(p-1) B] w(z)}{1+B w(z)} .
$$

And the above condition is equivalent to

$$
\left|\frac{\left(1+\frac{z f^{\prime \prime}(z)}{f^{\prime}(z)}-p\right)}{p b(A-B)-B\left(1+\frac{z f^{\prime \prime}(z)}{f^{\prime}(z)}-p\right)}\right|<1,
$$

holds true for some $b \neq 0$ complex, $p \in N,-1 \leq B<A \leq 1$, and for all $z \in U$.

It is noticed that, by giving specific values to $b, p, A$ and $B$, we obtain the following important subclasses studied by various authors in earlier works:

(i) $C(1,1,1,-1)=C$ is the well known class of convex functions;

(ii) $C((1-\alpha), 1,1,-1)=C(\alpha)$ (Robertson [11], and Pinchuk [10]);

(iii) $C\left((1-\alpha) \cos \lambda e^{-1 \lambda}, 1,1,-1\right)=C^{\lambda}(\alpha)$ (Chichra [2] and Sizuk [13]);

(iv) $C(b, 1,1,-1)=C(b)$ (Wiatrowski [14], Nasr and Aouf [6], [7]);

(v) $C(1,1, A, B)=C^{*}(A, B)$ (Mazur [5] and Silverman and Siliva [12]);

(vi) $C\left(\cos \lambda e^{-1 \lambda}, 1, A, B\right)=C^{\lambda}(A, B)$, where $C^{\lambda}(A, B)$ represents the class of functions $f(z) \in S$ satisfying the condition

$$
1+\frac{z f^{\prime \prime}(z)}{f^{\prime}(z)}=\frac{1+\left[B+(A-B) e^{-1 \lambda} \cos \lambda\right] w(z)}{1+B w(z)}
$$

where $w(z) \in \Omega,|\lambda|<\frac{\pi}{2}$, and $-1 \leq B<A \leq 1$; 
(vii) $C(1, p, 1,-1)=C(p)$, is the class of $p$-valent convex functions considered by Goodman [4];

(viii) $C(b, p, 1,-1)=C(b, p)$ (Aouf $[1])$;

(ix) $C(b, 1, A, B)=C(b, A, B)$; where $C(b, A, B)$ represents the class of functions $f(z) \in S$ satisfing the condition

$$
1+\frac{1}{b} \frac{z f^{\prime \prime}(z)}{f^{\prime}(z)}=\frac{1+A w(z)}{1+B w(z)}
$$

where $w(z) \in \Omega, b \neq 0$ complex, and $-1 \leq B<A \leq 1$.

In this paper we determine representation formulas, sharp coefficient estimates for the class $C(b, p, A, B)$ and we maximize $\left|a_{p+2}-\mu a_{p+1}^{2}\right|$ over the class $C(b, p, A, B)$. Also, we get sufficient condition for a function to be in the class $C(b, p, A, B)$, and sharp radius of convexity for the class $C(b, p, A, B)$.

2. Representation formulas for the class $C(b, p, A, B)$

We shall require the following lemmas in our investigation:

Lemma 1. Let the function $w(z)$ defined by

$$
w(z)=\sum_{k=1}^{\infty} c_{k} z^{k}
$$

be in the class $\Omega$. Then

$$
\left|c_{1}\right| \leq 1
$$

LEMMA 2. Let the function $f(z)$ be defined by (1.1). Then $f(z) \in C(b, p, A, B)$, $p \in N$ if and only if it can be represented as follows

$$
f^{\prime}(z)=p z^{p-1}\left[h^{\prime}(z)\right]^{p}(z \in U)
$$

for some $h(z) \in C(b, A, B)$.

P roof. By direct computation, we find from representation (2.4) that

$$
p+\frac{1}{b}\left(1+\frac{z f^{\prime \prime}(z)}{f^{\prime}(z)}-p\right)=p\left(1+\frac{1}{b} \frac{z h^{\prime \prime}(z)}{h^{\prime}(z)}\right) .
$$

Now the assertion (2.4) follows from (2.5) in view of definition (2.4).

LEMMA 3. Let the function $f(z)$ be defined by (1.1). Then $f(z) \in C(b, p, A, B)$, $p \in N$ if and only if it can be represented as follows

$$
f^{\prime}(z)=p z^{p-1}\left[g^{\prime}(z)\right]^{p b} \quad(z \in U),
$$

for some $g(z) \in C(1,1, A, B) \equiv C^{*}(A, B)$. 
Proof. By direct computation, we find from representation (2.6) that

$$
p+\frac{1}{b}\left(1+\frac{z f^{\prime \prime}(z)}{f^{\prime}(z)}-p\right)=p\left(1+\frac{z g^{\prime \prime}(z)}{g^{\prime}(z)}\right) .
$$

Now the assertion (2.6) follows from (2.7) in view of Definition 1.

3. Coefficient estimates for the class $C(b, p, A, B)$

We need in our investigation this lemma:

Lemma 4. Let the function $w(z)$ defined by $(2.1)$ be in the class $\Omega$. Then

$$
\left|c_{2}-\mu c_{1}^{2}\right| \leq \max \{1,|\mu|\},
$$

for any complex number $\mu$. Equality in (2.8) may be attained with the functions $w(z)=z^{2}$ and $w(z)=z$ for $|\mu|<1$ and $|\mu| \geq 1$, respectively.

THEOREM 1. If a function $f(z)$ defined by (1.1) is in the class $C(b, p, A, B)$ and if $\mu$ is any complex number, then

$$
\begin{aligned}
& \left|a_{p+2}-\mu a_{p+1}^{2}\right| \\
\leq & \frac{(A-B) p^{2}|b|}{2(p+2)} \max \left\{1,\left|[(A-B) p b-B]-\frac{2(A-B)(p+2) p^{2} b}{(p+1)^{2}} \mu\right|\right\} .
\end{aligned}
$$

The result (3.1) is sharp for each $\mu$.

Proof. Since $f(z) \in C(b, p, A, B)$, then from (1.7), we have

$$
w(z)=\frac{(p-1) f^{\prime}(z)-z f^{\prime \prime}(z)}{B z f^{\prime \prime}(z)-[(p-1) B+(A-B) p b] f^{\prime}(z)},
$$

and applying the definition (1.1), it can be shown that

$$
\begin{aligned}
& w(z)=\frac{(p+1)}{(A-B) p^{2} b} a_{p+1} z \\
& +\frac{1}{(A-B) p^{2} b}\left[2(p+2) a_{p+2}-\frac{(p+1)^{2}[(A-B) p b-B]}{(A-B) p^{2} b} a_{p+1}^{2}\right] z^{2}+\ldots
\end{aligned}
$$

Now compare the coefficients of $z$ and $z^{2}$ on both sides of (3.3), using the definition (2.1). We thus obtain

$$
c_{1}=\frac{(p+1)}{(A-B) p^{2} b} a_{p+1},
$$

and

$$
c_{2}=\frac{2(p+2)}{(A-B) p^{2} b} a_{p+2}-\frac{(p+1)^{2}[(A-B) p b-B]}{(A-B)^{2} p^{4} b^{2}} a_{p+1}^{2} .
$$

Consequently, we have

$$
a_{p+1}=\frac{(A-B) p^{2} b}{(p+1)} c_{1}
$$


and

$$
a_{p+2}=\frac{(A-B) p^{2} b}{2(p+2)} c_{2}+\frac{(p+1)^{2}[(A-B) p b-B]}{2(A-B)(p+2) p^{2} b} a_{p+1}^{2}
$$

Using (2.8), (3.6) and (3.7), we readily obtain (3.1).

Finally, the assertion (3.1) of Theorem 1 is sharp in view of the fact that the assertion (2.8) of Lemma 4 is sharp.

Corollary 1. If the function $f(z)$ defined by $(1.1)$ is in $C(b, p, A, B)$, then

$$
\left|a_{p+1}\right| \leq \frac{(A-B) p^{2}|b|}{(p+1)}
$$

and

$$
\left|a_{p+2}\right| \leq \frac{(A-B) p^{2}|b|}{2(p+2)} \max \{1,|(A-B) p b-B|\}
$$

The bounds in (3.8) and (3.9) are attained by the function $f^{*}(z)$ defined by

$$
f^{*^{\prime}}(z)=p z^{p-1}(1+B z)^{\frac{p(A-B) b}{B}}(B \neq 0) \text {. }
$$

Proof. The assertions (3.8) and (3.9) of Corollary 1 follow directly from (3.6) and (3.1), respectively.

We shall require the following Lemma in our investigation:

LEMMA 5. If $h(z)=z+\sum_{k=2}^{\infty} b_{k} z^{k} \in C(b, A, B)$, then

$$
\left|b_{2}\right| \leq \frac{(A-B)|b|}{2}
$$

and

$$
\left|b_{3}\right| \leq \frac{(A-B)|b|}{6} \max \{1,|(A-B) b-B|\} .
$$

The bounds in (3.11) and (3.12) are attained by the function $h^{*}(z)$ defined by

$$
h^{*^{\prime}}(z)=(1+B z)^{\frac{(A-B) b}{B}}(B \neq 0) \text {. }
$$

The bounds on the modulus of the second and third coefficients for functions in the class $C(b, p, A, B)$ are attained by another method as follows:

THEOREM 2. Let the function $f(z)$ defined by (1.1) be in $C(b, p, A, B)$, then

$$
\left|a_{p+1}\right| \leq \frac{(A-B) p^{2}|b|}{(p+1)}
$$

and 
(3.15) $\left|a_{p+2}\right| \leq \frac{(A-B) p^{2}|b|}{2(p+2)}\{\max \{1,|(A-B) b-B|\}+(A-B)(p-1)|b|\}$.

These results are sharp with equality for $f^{*}(z)$ defined by (3.10).

Proof. By Lemma 2, there exists an $h(z)=z+\sum_{k=2}^{\infty} b_{k} z^{k} \in C(b, A, B)$ such that

$$
f^{\prime}(z)=p z^{p-1}+\sum_{k=p+1}^{\infty} k a_{k} z^{k-1}=p z^{p-1}\left[1+\sum_{k=z}^{\infty} k b_{k} z^{k-1}\right]^{p-\alpha} .
$$

Expanding the right hand side of (3.16), we obtain

$$
f^{\prime}(z)=p z^{p-1}+2 p^{2} b_{2} z^{p}+p\left(3 p b_{3}+2 p(p-1) b_{2}^{2}\right) z^{p+1}+\ldots
$$

Equating coefficients from (2.3.16) and (2.3.17), we have

$$
\begin{gathered}
(p+1) a_{p+1}=2 p^{2} b_{2}, \\
(p+2) a_{p+2}=p\left(3 p b_{3}+2 p(p-1) b_{2}^{2}\right) .
\end{gathered}
$$

Thus, the result follows from Lemma 5 .

Remarks on Corollary 1 and Theorem 2. Comparing the results in Corollary 1 and Theorem 2 we see that:

(1) When $\max \{1,|(A-B) b-B|\}$ in Theorem 2 is $|(A-B) b-B|$, then Corollary 1 is a better result.

(2) When $\max \{1,|(A-B) b-B|\}$ in Theorem 2 is 1 , then Theorem 2 is a better result.

LEMMA 6. If $p$ and $m$ are positive integers, $b \neq 0$ complex and $-1 \leq B<$ $A \leq 1$, then

$$
\begin{aligned}
\prod_{j=0}^{m-1} & \frac{|(A-B) p b-B j|^{2}}{(j+1)^{2}} \\
= & \frac{1}{m^{2}}\left\{(A-B)^{2} p^{2}|b|^{2}\right. \\
& +\sum_{k=1}^{m-1}\left[(A-B)^{2} p^{2}|b|^{2}-2 k B p(A-B) \operatorname{Re}\{b\}+k^{2}\left(B^{2}-1\right)\right] \\
& \left.\times \prod_{j=0}^{k-1} \frac{|(A-B) p b-B j|^{2}}{(j+1)^{2}}\right\} .
\end{aligned}
$$

Proof. For $m=1$, the assertion (3.20) is obvious. Thus, in order to prove Lemma 6 by induction on $m$, suppose that the result (3.20) holds true for 
$m=q-1(q=1,2,3, \ldots)$. Then, observing that

$$
\frac{1}{q^{2}}\left\{(A-B)^{2} p^{2}|b|^{2}+\sum_{k=1}^{m-1}\left[(A-B)^{2} p^{2}|b|^{2}-2 k B p(A-B) \operatorname{Re}\{b\}\right.\right.
$$

$$
\left.\left.+k^{2}\left(B^{2}-1\right)\right] \prod_{j=0}^{k-1} \frac{|(A-B) p b-B j|^{2}}{(j+1)^{2}}\right\}
$$

$$
\begin{aligned}
= & \frac{1}{q^{2}}\left\{(A-B)^{2} p^{2}|b|^{2}+\sum_{k=1}^{q-2}\left[(A-B)^{2} p^{2}|b|^{2}-2 k B p(A-B) \operatorname{Re}\{b\}\right.\right. \\
& \left.\left.+k^{2}\left(B^{2}-1\right)\right] \prod_{j=0}^{k-1} \frac{|(A-B) p b-B j|^{2}}{(j+1)^{2}}\right\} \\
& +\left[(A-B)^{2} p^{2}|b|^{2}+(q-1)^{2}\left(B^{2}-1\right)-2(q-1) B p(A-B) \operatorname{Re}\{b\}\right] \times \\
& \times \prod_{j=0}^{q-2} \frac{|(A-B) p b-B j|^{2}}{(j+1)^{2}} \\
= & \left(\frac{q-1}{q}\right)^{2} \prod_{j=0}^{q-2} \frac{|(A-B) p b-B j|^{2}}{(j+1)^{2}} \\
& +\frac{\left[(A-B)^{2} p^{2}|b|^{2}-2(q-1) B p(A-B) \operatorname{Re}\{b\}+(q-1)^{2}\left(B^{2}-1\right)\right]}{q^{2}} \\
& \times \prod_{j=0}^{q-2} \frac{|(A-B) p b-B j|^{2}}{(j+1)^{2}} \\
= & \prod_{j=0}^{q-2} \frac{|(A-B) p b-B j|^{2}}{(j+1)^{2}} \cdot \frac{|(A-B) p b-(q-1) B|^{2}}{q^{2}} \\
= & \prod_{j=0}^{q-1} \frac{|(A-B) p b-B j|^{2}}{(j+1)^{2}},
\end{aligned}
$$

we conclude that the result (3.20) is valid also for $m=q$. This evidently completes the proof of Lemma 6 .

TheOREM 3. Let the function $f(z)$ defined by $(1.1)$ be in $C(b, p, A, B)$. If

$$
(A-B)^{2} p^{2}|b|^{2}+k^{2}\left(B^{2}-1\right)>2 k B P(A-B) \operatorname{Re}\{b\},
$$


then

$$
\left|a_{n}\right| \leq \frac{p}{n} \cdot \prod_{k=0}^{n-p-1} \frac{|(A-B) p b-B k|}{(k+1)} .
$$

The bounds in (3.23) are sharp for all admissible b, $p, A$ and $B$, and for each integer $n \geq p+1$.

Proof. Since $f(z) \in C(b, p, A, B),(1.7)$ gives

$$
\begin{gathered}
\left\{B z f^{\prime \prime}(z)-[B(p-1)+(A-B) p b] f^{\prime}(z)\right\} w(z) \\
=(p-1) f^{\prime}(z)-z f^{\prime \prime}(z), \quad w \in \Omega .
\end{gathered}
$$

Rewriting (3.24) in the form

$$
\begin{gathered}
\left\{B\left[p(p-1) z^{p-1}+\sum_{k=1}^{\infty}(p+k)(p+k-1) a_{p+k} z^{p+k-1}\right]-\right. \\
\left.-[B(p-1)+(A-B) p b] \cdot\left[p z^{p-1}+\sum_{k=1}^{\infty}(p+k) a_{p+k} z^{p+k-1}\right]\right\} w(z) \\
=(p-1)\left[p z^{p-1}+\sum_{k=1}^{\infty}(p+k) a_{p+k} z^{p+k-1}\right] \\
-\left[p(p-1) z^{p-1}+\sum_{k=1}^{\infty}(p+k)(p+k-1) a_{p+k} z^{p+k-1}\right]
\end{gathered}
$$

or, equivalently,

$$
\begin{gathered}
\left\{(A-B) p^{2} b+\sum_{k=1}^{\infty}(-B k+(A-B) p b)(p+k) a_{p+k} z^{k}\right\} w(z) \\
=-\sum_{k=1}^{\infty} k(p+k) a_{p+k} z^{k}
\end{gathered}
$$

we find that

$$
\sum_{k=0}^{\infty}\left\{[-B k+(A-B) p b](p+k) a_{p+k} z^{k}\right\} w(z)=-\sum_{k=0}^{\infty} k(p+k) a_{p+k} z^{k}
$$

where $a_{p}=1$ and $w(z)$ is given, as before, by (2.1).

Equating the coefficients of $z^{m}$ on both sides of (3.27), we obtain

$$
\sum_{k=0}^{m-1}\left[(-B k+(A-B) p b) a_{p+k} c_{m-k}=-m(p+m) a_{p+m},\right.
$$


which shows that $a_{p+m}$ on the right-hand side depends only on the coefficients

$$
a_{p}, a_{p+1}, \ldots, a_{p+m-1} .
$$

Occuring on the left-hand side. Hence we can write

$$
\begin{array}{r}
\left.\sum_{k=0}^{m-1}\{[-B k+(A-B) p b)(p+k)] a_{p+k} z^{k}\right\} w(z) \\
=\sum_{k=0}^{m} k(p+k) a_{p+k} z^{k}+\sum_{k=m+1}^{\infty} A_{k} z^{k},
\end{array}
$$

for $m=1,2,3, \ldots$, and for a proper choice of $A_{k}(k \geq 0)$.

Let $z=r e^{i \theta}, 0<r<1,0 \leq \theta \leq 2 \pi$. Then

$$
\begin{aligned}
& \sum_{k=0}^{m-1}|[-B k+(A-B) p b(p+k)]|^{2}\left|a_{p+k}\right|^{2} r^{2 k} \\
= & \frac{1}{2 \pi} \int_{0}^{2 \pi}\left|\sum_{k=0}^{m-1}\{[-B k+(A-B) p b](p+k)\} a_{p+k} r^{k} e^{i k \theta}\right|^{2} d \theta \\
\geq & \frac{1}{2 \pi} \int_{0}^{2 \pi}\left|\sum_{k=0}^{m-1}\{[-B k+(A-B) p b](p+k)\} a_{p+k} r^{k} e^{i k \theta}\right|^{2}\left|w\left(r e^{i \theta}\right)\right|^{2} d \theta \\
\geq & \frac{1}{2 \pi} \int_{0}^{2 \pi}\left|\sum_{k=0}^{m} k(p+k) r^{k} e^{i k \theta}+\sum_{k=m+1}^{\infty} A_{k} r^{k} e^{i k \theta}\right|^{2} d \theta \\
\geq & \sum_{k=0}^{m} k^{2}\left|a_{p+k}\right|^{2} r^{2 k}+\sum_{k=m+1}^{\infty}\left|A_{k}\right|^{2} r^{2 k} \geq \sum_{k=0}^{m} k^{2}\left|a_{p+k}\right|^{2} r^{2 k}
\end{aligned}
$$

Letting $r \rightarrow 1$ in (3.30), we obtain

$$
\begin{gathered}
\sum_{k=0}^{m-1}\left[|(-B k+(A-B) p b)(p+k)|^{2}-k^{2}(p+k)^{2}\right]\left|a_{p+k}\right|^{2} \\
\geq m^{2}(p+m)^{2}\left|a_{p+m}\right|^{2} .
\end{gathered}
$$

Setting $m=n-p$ in (3.31), we are led finally to the inequality

$$
\begin{array}{r}
n^{2}(n-p)^{2}\left|a_{n}\right|^{2} \leq(A-B)^{2} p^{4}|b|^{2} \\
+\sum_{k=1}^{n-p-1}\left[|(-B k+(A-B) p b)(p+k)|^{2}-k^{2}(p+k)^{2}\right]\left|a_{p+k}\right|^{2} \\
\quad(n=p+1, p+2, p+3, \ldots) .
\end{array}
$$


Then, for $n=p+1,(3.3)$ immediately yields

$$
\left|a_{p+1}\right| \leq \frac{(A-B) p^{2}|b|}{(p+1)}
$$

which proves (3.24) for $n=p+1$.

To establish (3.23) for $n>p+1$, we use an induction argument. Then

$$
\begin{aligned}
\left.a_{n}\right|^{2} & \leq \frac{p^{2}}{n^{2}(n-p)^{2}}\left\{(A-B)^{2} p^{2}|b|^{2}+\right. \\
& +\sum_{k=1}^{n-(p+1)}\left[(A-B)^{2} p^{2}|b|^{2}-2 k B p(A-B) \operatorname{Re}\{b\}+k^{2}\left(B^{2}-1\right)\right] \\
& \left.\times \prod_{j=0}^{k-1} \frac{|(A-B) p b-B j|^{2}}{(j+1)^{2}}\right\} .
\end{aligned}
$$

Thus from (3.32), (3.34) and Lemma 2.3.1 with $m=n-p$, we obtain

$$
\left|a_{n}\right|^{2} \leq \frac{p^{2}}{n^{2}} \cdot \prod_{j=0}^{n-p-1} \frac{|(A-B) p b-B j|^{2}}{(j+1)^{2}} .
$$

This completes the proof of (3.23). This proof is based on a technique found in Clunie [3].

\section{A sufficient condition for the class $C(b, p, A, B)$}

We now establish a sufficient condition for a function to be in the class $C(b, p, A, B)$.

THEOREM 4. Let the function $f(z)$ defined by (1.1) be analytic in $U$. Then $f(z) \in C(b, p, A, B)$ if, for $b \neq 0$ complex, $p \in N$, and $1 \leq B<A \leq 1$,

$$
\sum_{n=p+1}^{\infty} \frac{n}{p}\{(n-p) B \mid\}\left|a_{n}\right| \leq p(A-B)|b| .
$$

Proof. Let $|z|=r<1$. Noting that

$$
\left|z f^{\prime \prime}(z)-(p-1) f^{\prime}(z)\right|<\sum_{n=p+1}^{\infty} n(n-p)\left|a_{n}\right| r,
$$


and

$$
\begin{aligned}
& \left|p b(A-B) f^{\prime}(z)-B\left[z f^{\prime \prime}(z)-(p-1) f^{\prime}(z)\right]\right| \\
& \quad>\left\{p^{2}|b|(A-B)-\sum_{n=p+1}^{\infty} n|p b(A-B)-(n-p) B| a_{n} \mid\right\} r
\end{aligned}
$$

we see that

$$
\begin{aligned}
& \left|z f^{\prime \prime}(z)-(p-1) f^{\prime}(z)\right|-\left|p b(A-B) f^{\prime}(z)-B\left[z f^{\prime \prime}(z)-(p-1) f^{\prime}(z)\right]\right| \\
< & \left\{\sum_{n=p+1}^{\infty} n\{(n-p)+|p b(A-B)-(n-p) B|\}\left|a_{n}\right|-p^{2}(A-B)|b|\right\} r .
\end{aligned}
$$

The right-hand side of (4.4) is non-positive by $(4.1)$, so that $f(z) \in$ $C(b, p, A, B)$ by Definition 1 .

5. Radius of convexity for the class $C(b, p, A, B)$

THEOREM 5. The sharp radius of convexity of the class $C(b, p, A, B)$ is given by

$r_{c}= \begin{cases}2\left\{(A-B)|b| \sqrt{|b|^{2}(A-B)^{2}+4 B[B+(A-B) \operatorname{Re}\{b\}]}\right\}^{-1} & (B \neq 0), \\ \{A|b|\}^{-1} & (B=0) .\end{cases}$

(5.1) is real and finite only when $B \neq 0$ and such that

$$
|b|^{2}(A-B)^{2}+4 B[B+(A-B) \operatorname{Re}\{b\}]>0 .
$$

The bounds in (5.1) are sharp for the function $f^{*}(z)$ given by

$$
f^{*^{\prime}}(z)= \begin{cases}p z^{p-1}(1+B z)^{\frac{p b(A-B)}{B}} & (B \neq 0) \\ p z^{p-1} \exp \{p b A z\} & (B=0)\end{cases}
$$

Proof. Since $f(z) \in C(b, p, A, B)$, the condition (1.9) in conjunction with the Schwarz lemma [8] implies that

$$
\left|\frac{z f^{\prime \prime}(z)}{f^{\prime}(z)}-\zeta\right|<R
$$

where

$$
\zeta=\frac{(p-1)-B[(p-1) B+p b(A-B)] r^{2}}{1-B^{2} r^{2}}
$$

and

$$
R=\frac{p|b|(A-B) r}{1-B^{2} r^{2}} \quad(|z|=r)
$$

Hence we have 


$$
\begin{aligned}
& \frac{p-p|b|(A-B) r-B[p B+p \operatorname{Re}\{b\}(A-B)] r^{2}}{1-B^{2} r^{2}} \\
\leq & \operatorname{Re}\left\{1+\frac{z f^{\prime \prime}(z)}{f^{\prime}(z)}\right\} \leq \frac{p+p|b|(A-B) r-B[p B+p \operatorname{Re}\{b\}(A-B)] r^{2}}{1-B^{2} r^{2}} .
\end{aligned}
$$

From the first part of the inequalities in (5.4), it follows readily that

$$
\operatorname{Re}\left\{1+\frac{z f^{\prime \prime}(z)}{f^{\prime}(z)}\right\}>0 \quad \text { for }|z|<r_{c}
$$

where $r_{c}$ is given by (5.1), provided that the condition (5.2) is satisfied. To show that the result (5.1) is sharp, we let $f^{*^{\prime}}(z)$ be given (5.3), and put

$$
\zeta= \begin{cases}-\left(\frac{B r^{2}+\sqrt{\frac{\bar{b}}{b}} r}{1+B \sqrt{\frac{\bar{b}}{b}} r}\right) & B \neq 0, \\ -\left(\sqrt{\frac{\bar{b}}{b}} r\right) & B=0 .\end{cases}
$$

We thus obtain

$$
1+\frac{\zeta f^{*^{\prime \prime}}(\zeta)}{f^{*^{\prime}}(\zeta)}= \begin{cases}\frac{p-p(A-B)|b| r-p B[B+(A-B) b] r^{2}}{1-B^{2} r^{2}}, & B \neq 0 \\ p(1-A|b| r), & B=0\end{cases}
$$

which obviously has a zero real part when $r$ is given by (5.1). This completes the proof of Theorem 5 .

\section{References}

[1] M. K. Aouf, P-valent classes related to convex functions of complex order, Rocky Mountain J. Math. 12 (1985), no.4, 853-863.

[2] P. N. Chichra, Regular functions $f(z)$ for which $z f^{\prime}(z)$ is $\theta$-spirallike, Proc. Amer. Math. Soc. 49 (1975), 151-160.

[3] J. Clunie, On meromorphic schlicht functions, J. London Math. Soc. 34 (1959), 215.

[4] A. W. Goodman, On the Schwarz-Christoffel transformation and p-valent functions, Trans. Amer. Math. Soc. 68 (1950), 204-223.

[5] R. Mazur, On a subclass of convex function, Zeszyty Nauk. Politech. Lódź. Mat. Nr. 353, Z. 13 (1981), 15-19.

[6] M. A. Nasr and M. K. Aouf, On convex functions of complex order, Bull. Fac. Sci. Mansoura Univ. 9 (1982), 565-582.

[7] M. A. Nasr and M. K. Aouf, Radius of convexity for the class of starlike functions of complex order, Bull. Fac. Sci. Assiut Univ. A(12) (1983), no. 1, 153-159.

[8] Z. Nehari, Conformal Mapping, McGraw-Hill, New York. 1952. 
[9] D. A. Patil and N. K. Thakare, On coefficient bounds of p-valent $\lambda$-spiral functions of order $\alpha$, Indian J. Pure Appl. Math. 10 (1979), 842-853.

[10] B. Pinchuk, On starlike and convex functions of order $\alpha$, Duke Math. J. 35 (1968), 721-734.

[11] M. S. Robertson, On the theory of univalent functions, Ann. Math. 37 (1936), 374-408.

[12] H. Silverman and E. M. Silvia, Subclasses of starlike functions subordinate to convex functions, Canad. J. Math. 37 (1985), no. 1, 48-61.

[13] P. I. Sizuk, Regular functions $f(z)$ for which $z f^{\prime}(z)$ is $\theta$-spiral shaped of order $\alpha$, Sibrisk, Mat. Z. 16 (1975), 1286-1290, 1371.

[14] P. Wiatrowski, The coefficients of a certain family of holomorhic functions, Zeszyty Nauk. Univ. Lódź. Mat. 39 (1971), 75-85.

DEPARTMENT OF MATHEMATICS

FACULTY OF SCIENCE

MANSOURA UNIVERSITY

MANSOURA, EGYPT

E-mail: sinfac@mum.mans.eun.eg

Received July 12, 1999. 
